\title{
Finite Difference Approximation for Space Fractional Soil Moisture Diffusion Equation and Its Application
}

\author{
S. M. Jogdand ${ }^{1}$, K .C. Takale ${ }^{2}$, A. S. Jagtap ${ }^{3}$ \\ 1. Department of Mathematics, Shri Sant Gadge Maharaj Mahavidyalya, Loha, (Maharashtra), India \\ 2. Department of Mathematics, RNC Arts, JDB Commerce and NSC Science College, Nashik-Road, \\ (Maharashtra), India. \\ 3. Department of Mathematics, Tuljaram Chaturchand College, Baramati, (Maharashtra), India.
}

\begin{abstract}
The purpose of this paper is to develop the space fractional order explicit finite difference scheme for fractional order soil moisture diffusion equation with the initial and boundary conditions. We prove that the solution of the space fractional order finite difference scheme is conditionally stable and the convergence of the scheme is discussed at the length. Also as an application of this scheme, numerical solution for space fractional soil moisture diffusion equation is obtained and it is represented graphically by the software 'Mathematica'.
\end{abstract}

Keywards: finite difference, explicit, diffusion equation, soil moisture.

\section{Introduction}

Recently we found new applications of fractional calculus in science, engineering, finance and hydrology $[2,3,7,9,10]$. A physical or mathematical approach to analomous diffusion is based on generalized diffusion equation containing fractional order derivatives in time or space or space-time. Now a day, fractional diffusion equations have been studied by many mathematicians and researchers $[1,4,11,12]$. In this study we develop the space fractional order explicit finite difference scheme for fractional order soil moisture diffusion equation.

Most physical phenomenon be involved using partial differential equation. The hydrodynamics of the water into soil is a very complex phenomenon and infiltration is continuous to occupy the attention of soil physicists and engineers. A theoretical and experimental analysis is made to understand this phenomenon by many soil Scientists from the beginning and birth of soil Physics $[5,6,8]$. Furthermore, in numbers of laboratory and field experiments where the results were only qualitatively interpreted and in the real application of physical theories to the solution of transport processes in soil, to tillage and compaction of soil etc.s

First experimental study on the movement of water in the soil was done by H. Darcy (1856). Edgar Buckingham (1907) described the water flow in unsaturated porous media modifying the equation of Darcy. Richard's (1931) combined the equation and Buckingham with the equation of continuity to establish an over all relationship.

Consider the general diffusion equation of unsaturated flow of soil moisture as follows,

$$
\frac{\partial}{\partial x}\left(D \frac{\partial \theta}{\partial x}\right)+\frac{\partial}{\partial y}\left(D \frac{\partial \theta}{\partial y}\right)+\frac{\partial}{\partial z}\left(D \frac{\partial \theta}{\partial z}\right)+\frac{d K}{d z}=\frac{\partial \theta}{\partial t}
$$

where,

$$
\begin{aligned}
\theta(x, y, z, t) & =\text { the volumetric soil moisture content, } \\
D & =\text { the diffusivity of soil moisture, } \\
D & =D(\theta) \text { is a function of moisture content and } \\
K & =\text { the capillary or hydraulic conductivity of soil moisture. }
\end{aligned}
$$

If the flow is considered in $x$ direction and assume that $D$ is constant then equation (1.1) becomes

$$
\frac{\partial \theta}{\partial t}=D \frac{\partial^{2} \theta}{\partial z^{2}}
$$

Which is the diffusion heat flow equation, studied by Richard's for water flow instead of heat flow. Then the model problem is

$$
\frac{\partial \theta}{\partial t}=D \frac{\partial^{2} \theta}{\partial x^{2}}, t>0, x \geq 0
$$


We solve the particular problem of moisture flow in to a horizontal tube, we impose proper initial and boundary conditions.For that with an initial uniform moisture percentage of

$\theta$ is $\theta_{0}$ ( $\theta_{0}$ is constant) and for which at time $t=0$, become initial condition and which is mathematically expressed as follows

$$
\theta(x, t)=\theta_{0}, t=0, x \geq 0
$$

For left boundary condition, there is applied a source of water placed at $x=0$ so as to maintain at all times after $t=0$ is $\theta_{L}$, and which is mathematically expressed as ,

$$
\theta(x, t)=\theta_{L}, x=0, t \geq 0
$$

For right boundary condition, there is applied a source of water placed at semi-infinite plane so as to maintain at all times after $t=0$ is $\theta_{R}$, and which is mathematically expressed as,

$$
\theta(x, t)=\theta_{R}, x \rightarrow \infty, t \geq 0
$$

There fore, we have the model IBVP for soil moisture flow which is given as follows

$$
\frac{\partial \theta}{\partial t}=D \frac{\partial^{2} \theta}{\partial x^{2}}, t>0, x \geq 0
$$

Subject to the initial and boundary conditions

$$
\begin{aligned}
& \theta(x, t)=\theta_{0}, t=0, x \geq 0 \\
& \theta(x, t)=\theta_{L}, x=0, t \geq 0, \theta(x, t)=\theta_{R}, x \rightarrow \infty, t \geq 0
\end{aligned}
$$

Where $\theta(x, t)$ is volumetric water content and $D$ is the diffusivity constant of soil moisture.

In the next section we develop the explicit finite difference scheme (SFEFDS) for space fractional soil moisture diffusion equation.

The plan of the paper is as follows: In section 2, the fractional order explicit finite difference scheme is developed for space fractional soil moisture diffusion equation. The section 3 is devoted for stability of the scheme and the question of convergence is proved in section 4 . The numerical solution of space fractional soil moisture diffusion equation is obtained using Mathematica software in the section 5 .

\section{Finite Difference Scheme:}

Consider the space fractional soil moisture diffusion equation (SFSMDE) with initial and boundary conditions as follows.

$$
\begin{gathered}
\frac{\partial \theta(x, t)}{\partial t}=D \frac{\partial^{\alpha} \theta(x, t)}{\partial x^{\alpha}}, t>0, x_{L} \leq x \leq x_{R}, 1<\alpha \leq 2 \\
\text { Initial condition: } \theta(x, 0)=\theta_{0}, \\
\text { boundary conditions: }(0, t)=\theta_{L},: \theta_{x}(x, t)=\theta_{R}, x \rightarrow \infty, t \geq 0
\end{gathered}
$$

where the diffusion coefficient $D>0$, and $\frac{\partial^{\alpha} \theta(x, t)}{\partial x^{\alpha}}={ }_{0} D_{x}^{\alpha} \theta(x)$ is Capiuto's fractional derivative, it is defined as [10]

$$
\frac{\partial^{\alpha} \theta}{\partial x^{\alpha}}={ }_{0} D_{x}^{\alpha} \theta(x)= \begin{cases}\frac{d^{m} \theta(x)}{d x^{m}} & \alpha=M \in N \\ \frac{1}{\Gamma(M-\alpha)} \int_{0}^{x}(x-\xi)^{m-\alpha-1} \frac{d^{m} \theta(\xi)}{d \xi^{m}} d \xi & m-1<\alpha<m\end{cases}
$$

Where $\Gamma(\cdot)$ is the gamma function.

Consider $h=\frac{x}{k} ; k$ is positive integer, using second order difference approximation, we get 


$$
\begin{aligned}
& \frac{\partial^{\alpha} \theta(x, t)}{\partial x^{\alpha}}={ }_{0} D_{x}^{\alpha} \theta(x, t)=\frac{1}{\Gamma(2-\alpha)} \int_{0}^{x} \frac{1}{(x-\xi)^{\alpha-1}} \frac{\partial^{2} \theta(\xi)}{\partial \xi^{2}} d \xi \\
&=\frac{1}{\Gamma(2-\alpha)} \sum_{j=0}^{k=1} \int_{j h}^{(i+1) h} \eta^{1-\alpha} \frac{\partial^{2} \theta(x-\eta, t)}{\partial \eta^{2}} d \eta
\end{aligned}
$$

Put $\eta=x-\xi, d \eta=-d \xi, \xi=x-\eta$

$$
\begin{aligned}
& \cong \frac{1}{\Gamma(2-\alpha)} \sum_{j=0}^{k-1} \frac{\theta(x-(j-1) h, t)-2 \theta(x-j h, t)+\theta(x-(j+1) h, t)}{h^{2}} \int_{j h}^{(i+1) h} \eta^{1-\alpha} d \eta \\
& =\frac{h^{-\alpha}}{\Gamma(3-\alpha)} \sum_{j=0}^{k-1}[\theta(x-(j-1) h, t)-2 \theta(x-j h, t)+\theta(x-(j+1), t)]\left[(j+1)^{2-\alpha}-j^{2-\alpha}\right]
\end{aligned}
$$

Let $\Delta t=\tau>0$ be the grid step in time, $t_{n}=n \Delta t=n \tau, 0 \leq t_{n} \leq T, \Delta x=h>0$ be the grid step in space

$$
\begin{aligned}
& x_{j}=i h, j-0,1,2, \ldots, N \\
& \therefore N h=L \\
& \therefore N=\frac{L}{h}
\end{aligned}
$$

Let $\theta_{0}^{n}=\theta(0, n \tau), \theta_{1}^{n}=\theta(h, n \tau), \ldots \theta_{k-j}^{n}=\theta((k-j) h, n \tau), \ldots, \theta_{k}^{n}=\theta(k h, n \tau)$

We approximate the soil moisture space fractional diffusion equation (SMSFDE) (2.1) by using an explicit finite difference scheme (EFDS), we get

$$
\frac{\theta_{k}^{n+1}}{\tau}=\frac{D h^{-\alpha}}{\Gamma(3-\alpha)} \sum_{j=0}^{k-1}\left[\theta_{k-j+1}^{n}-2 \theta_{k-j}^{n}+\theta_{k-j-1}^{n}\right]\left[(j+i)^{2-\alpha}-j^{2-\alpha}\right]
$$

After simplification we get

$$
\theta_{k}^{n+1}=b_{k} \theta_{k+1}^{n}+(1-2 b k) \theta_{k}^{n}+b_{k} \theta_{k-1}^{n}+b_{k} \sum_{j=1}^{k-1} g_{j}\left[\theta_{k-j+1}^{n}-2 \theta_{k-j}^{n}+\theta_{k-j-1}^{n}\right]
$$

where $b_{k}=\frac{\tau D}{h^{\alpha} \Gamma(3-\alpha)}, g_{k}=(k+1)^{2-\alpha}-k^{2-\alpha}$.

The initial condition is approximated as

$$
\theta_{j}^{0}=\theta_{0}, j=1,2, \ldots, N .
$$

The left boundary condition is approximated as

$$
\theta_{0}^{n}=\theta_{L}, n=0,1,2, \ldots, N
$$

Now using central difference formula the right boundary condition is approximated as follows.

$$
\frac{\theta_{N+1}^{n}-\theta_{N-1}^{n}}{2 h}=0, n-0,1,2, \ldots, N .
$$

Therefore the fractional approximated initial boundary value problem (IBVP) is 


$$
\begin{gathered}
\theta_{k}^{n+1}=b_{k} \theta_{k+1}^{n}+\left(1-2 b_{k}\right) \theta_{k}^{n}+b_{k} \theta_{k-1}^{n}+b_{k} \sum_{j=1}^{k-1} g_{j}\left[\theta_{k-j+1}^{n}-2 \theta_{k-j}^{n}+\theta_{k-j-1}^{n}\right] \\
\quad \text { initial condition }: \theta_{j}^{0}=\theta_{0}, j=1,2, \ldots, N \\
\quad \text { boundary conditions }: \theta_{0}^{n}=\theta_{L}, n=0,1,2, \ldots, N
\end{gathered}
$$

where $b_{k}=\frac{\tau D}{h^{\alpha} \Gamma(3-\alpha)}, g_{k}=(k+1)^{2-\alpha}-k^{2-\alpha}$, where $k=1,2, \ldots, N$. .

Therefore the IBVP (2.7) - (2.9) can be written in the following matrix equation.

$$
U^{n+1}=A U^{n}+B
$$

where,

$$
U^{n}=\left(\theta_{1}^{n}, \theta_{2}^{n}, \ldots, \theta_{N}^{n}\right)^{\tau} .
$$

and $A=\left(a_{i j}\right)$ is a square matrix of coefficients of order $N$, where

$$
a_{i j}=\left\{\begin{array}{l}
0, \text { when } j \geq i+2 \\
b_{i}, \text { when } j=i+1 \\
1-b_{i}\left(2-g_{1}\right), \text { when } j=i=2,3,4, \ldots, N \\
b_{i}\left(1-2 g_{1}+g_{2}\right), \text { when } j=i-1, i=3,4, \ldots, N-1 \\
b_{i}\left(g_{i-j-1}-2 g_{i-j}+g_{i-j+1}\right), \text { when } j=i-2 .
\end{array}\right.
$$

while,

$$
\begin{aligned}
& a_{11}=1-2 b_{1} \\
& a_{21}=1-2 g_{1} \\
& a_{32}=1-2 g_{1}+g_{2} \text { and } \\
& a_{i 1}=b_{i}\left(g_{i-2}-2 g_{i-1}\right), 3 \leq i \leq N \\
& a_{N, N-1}=b_{N}\left(2-2 g_{1}+g_{2}\right) \text { and } \\
& B=\theta_{L}\left[b_{1} g_{0}, b_{2} g_{1}, \ldots, b_{N} g_{N-1}\right]^{\tau}
\end{aligned}
$$

The above system of algebraic equations is solved by using Mathematica software in section 5 .

\section{Stability:}

This section is devoted for the stability criteria of the space fractional explicit finite difference scheme (2.7) - (2.9) for the SFDE (2.1) - (2.3).

Theorem 3.1: The solution of the space fractional explicit finite difference scheme (SFEFDS) (2.7) - (2.9) for the SFDE (2.1) - (2.3) is conditionally stable.

Proof: Consider the equation (2.10), we have,

$$
U^{n+1}=A U^{n}+B
$$

By Gerschgorin's first theorem [6], let $\lambda_{i}$ be an eigenvalue of the matrix $A$ to linear system of equation (2.10), and $x$ be the corresponding eigenvector then $A x=\lambda x$. Choose $i$ such that $\left|x_{i}\right|=\max \left\{\left|x_{j}\right|, j=0,1,2, \ldots, N\right\}$, then $\sum_{j=0}^{N} a_{i j} x_{j}=\lambda x_{i}$, and therefore 


$$
\lambda=a_{i i}+\sum_{j=1 j \neq i}^{N-1} a_{i} \frac{x_{j}}{x_{i}}
$$

We substitute the value of $a_{i j}$ in equation (3.1), we get

(i) when $i=1$

(ii) when $i=2$

$$
\begin{aligned}
& \lambda=1-2 b_{1}+b_{1} \frac{x_{2}}{x_{1}}, \quad 1-b_{1} \leq 1 \\
& \Rightarrow|\lambda| \leq 1 \text { if } b_{1} \leq 1
\end{aligned}
$$

$$
\begin{aligned}
& \lambda=1-b_{2}\left(2-g_{1}\right)+b_{2}\left(1-2 g_{1}\right) \frac{x_{1}}{x_{0}}+b_{2} \frac{x_{2}}{x_{1}} \\
& \leq 1-b_{2} g_{1} \leq 1 \\
& \text { and } \lambda \geq 1-b_{2}\left(2-g_{1}\right)+b_{2}\left(1-2 g_{1}\right)-b_{2} \\
& \Rightarrow \lambda \geq-1 \text { if } b_{2} \leq \frac{1}{2} \\
& \therefore-1 \leq \lambda \leq 1 \text { if } b_{2} \leq \frac{1}{2} \\
& \Rightarrow|\lambda| \leq 1 \quad \text { if } b_{2} \leq \frac{1}{2}
\end{aligned}
$$

(iii) when $i=3$

(iv) when $4 \leq i \leq N-1$

$$
\begin{aligned}
& \lambda=1-b_{3}\left(2-g_{1}\right)+b_{3}\left(g_{1}-2 g_{2}\right) \frac{x_{1}}{x_{0}}+b_{3}\left(1-2 g_{1}+g_{2}\right) \frac{x_{2}}{x_{1}}+b_{3} \frac{x_{3}}{x_{2}} \\
& \leq 1 \text { and } \\
\lambda & \geq 1-b_{3}\left(2-g_{1}\right)-b_{3}\left(g_{1}-2 g_{2}\right)-b_{3}\left(1-2 g_{1}+g_{2}\right)-b_{3} \\
& \geq-1 \text { if } b_{3}\left(4-2 g_{1}-g_{2}\right) \leq 1 \\
|\lambda| & \leq 1 \text { if } b_{3}\left(4-2 g_{1}-g_{2}\right) \leq 1, b_{3} \leq \frac{1}{\left(4-2 g_{1}-g_{2}\right)}
\end{aligned}
$$

$$
\lambda=1-b_{i}\left(2-g_{1}\right)+b_{i} \frac{x_{i+1}}{x_{i}}+b_{i} \sum_{j=2}^{i-1}\left(g_{i-j-1}-2 g_{i-j}+g_{i-j+1}\right) \frac{x_{j}}{x_{i}}+b_{i}\left(g_{i-2}-2 g_{i-1}\right) \frac{x_{j}}{x_{i}}+b_{i}\left(1-2 g_{1}+g_{2}\right)
$$

$$
g_{i} \geq g_{i+1}>0, \quad g_{i-j-1}-2 g_{i-j}+g_{i-j+1}>0 \text { for } j=1,2, \ldots, i-1, i=1,2, \ldots, N-2
$$

Now $\sum_{j=2}^{i-1}\left(g_{i-j-1}-2 g_{i-j}+g_{i-j+1}\right) \frac{x_{i-2}}{x_{i}} \leq g_{i-1}-g_{i-2}+g_{0}-g_{1}$

Since $b_{i}{ }^{\prime} s$ are non-negative numbers, we get 
$\lambda \leq 1-b_{i}\left(2-g_{1}\right)+b_{i}+b_{i}\left(g_{i-1}-g_{i-2}+g_{0}-g_{1}\right)+b_{i}\left(g_{i-2}-2 g_{i-1}\right)+b_{i}\left(1-2 g_{1}-g_{2}\right)$

$\leq 1$ and

$\lambda \geq 1-b_{i}\left(2-g_{1}\right)-b_{i}-b_{i}\left(g_{i-1}-g_{i-2}+g_{0}-g_{1}\right)-b_{i}\left(g_{i-2}-2 g_{i-1}\right)-b_{i}\left(1-2 g_{1}-g_{2}\right)$

$\therefore|\lambda| \leq 1$ if $b_{i} \leq \frac{1}{\left(4+g_{2}+g_{0}\right)}$

(v) when $i=N$

$$
\begin{aligned}
\lambda & \leq 1-b_{N}\left(2-g_{1}\right)+b_{N}\left(g_{N-2}-2 g_{N-1}\right) \frac{x_{1}}{x_{N}}+b_{N} \sum_{j=2}^{i-1}\left(g_{i-j-1}-2 g_{i-j}+g_{i-j+1}\right) \frac{x_{j-2}}{x_{N}} \\
& +b_{N}\left(2-2 g_{1}+g_{2}\right) \\
& \leq 1 \text { and }
\end{aligned}
$$

$$
\begin{aligned}
& \lambda \geq 1-b_{N}\left(2-g_{1}\right)-b_{N}\left(g_{N-2}-2 g_{N-1}\right)-b_{N}\left(g_{N-1}-g_{N-2}+g_{0}-g_{1}\right)-b_{N}\left(2-2 g_{1}+g_{2}\right) \\
& -b_{N}\left(2-2 g_{1}+g_{2}\right) \\
& \geq-1 \\
& \text { If } b_{N} \leq \frac{1}{\left(4-4 g_{1}+g_{0}+g_{2}\right)} \text { then }-1 \leq \lambda \leq 1 \\
& \Rightarrow|\lambda| \leq 1 \text { if } b_{N} \leq \frac{1}{\left(4-4 g_{1}+g_{0}+g_{2}\right)}
\end{aligned}
$$

Therefore from (i) - (v) we have proved that if

$$
\max _{1 \leq i \leq N-1}\left\{b_{1}, b_{2}, b_{3}, b_{i}, b_{N}\right\} \leq 1
$$

then the spectral radius $\rho(A)$ of the matrix satisfies $\rho(A) \leq 1$.

If

$$
\max _{1 \leq i \leq N-1}\left\{b_{1}, b_{2}, b_{3}, b_{i}, b_{N}\right\} \leq 1
$$

then there exists a positive number $\varepsilon \leq c \tau$ such that

$$
\|A\|_{m} \leq \rho(A)+C \tau \leq 1+0(\tau)
$$

Therefore, this proves that the finite difference scheme is conditionally stable.

\section{Convergence:}

In this section we discuss the question of convergence. Consider another vector

$$
\bar{U}^{n}=\left\{\theta\left(x_{1}, t_{n}\right), \ldots, \theta\left(x_{j}, t_{n}\right), \ldots, \theta\left(x_{N}, t_{n}\right)\right\}^{\tau}
$$

Satisfying the finite difference scheme (10), becomes

$$
\bar{U}^{n+1}=A \bar{U}^{n}+B+\tau^{n}
$$

where $\tau^{n}$ is the vector of the truncation errors at time level $t_{n}$.

Theorem 4.1: If

$$
\max _{1 \leq i \leq N-1}\left\{b_{1}, b_{2}, b_{3}, b_{i}, b_{N}\right\} \leq 1
$$

then the fractional order explicit finite difference scheme (2.7) - (2.9) for SMSFDE (2.1) - (2.3) is conditionally convergent.

Proof: Let $\Omega$ be the region $\theta<x<L, 0<t<T$.

Take $\left(x_{j}, t_{n}\right)=(j \Delta x, n \Delta t)$ for $j=1,2, \ldots, N$ and $n=1,2, \ldots, M$ with $N \Delta x=L, M \Delta t=T$.

We introduce the vector 


$$
\bar{U}^{n}=\left(\theta\left(x_{1}, t_{n}\right), \ldots, \theta\left(x_{j}, t_{n}\right), \ldots, \theta\left(x_{N}, t_{n}\right)\right)^{\tau}
$$

satisfying the finite difference scheme (2.10), we get

$$
\bar{U}^{n+1}=A \bar{U}^{n}+B+\tau^{n}
$$

where $\tau^{n}$ is the vector of the truncation errors at level $t_{n}$

$$
\theta_{j}^{0}=\theta_{0}
$$

and

$$
-\theta_{0}^{n}=\theta_{L}, \frac{\theta_{N+1}^{n}-\theta_{N-1}^{n}}{2 h}=0
$$

Now, we substract (2.10) - (4.2), we get

$$
\bar{U}^{n+1}-U^{n+1}=A\left(\bar{U}^{n}-U^{n}\right)+\tau^{n}
$$

Clearly, ${ }^{n}$ satisfies (1.7), we have

$$
\begin{gathered}
E_{k}^{n+1}=b_{k} E_{k+1}^{n}+\left(1-2 b_{k}\right) E_{k}^{n}+b_{k} E_{k-1}^{n}+b_{k} \sum_{j=1}^{k-1} g_{j}\left[E_{k-j+1}^{n}-2 E_{k-j}^{n}+E_{k-j-1}^{n}\right]+\tau^{n} \\
E_{j}^{0}=0, E_{0}^{n}=0, E_{N}^{n}=0 \\
\text { As } \quad \max _{1 \leq i \leq N-1}\left\{b_{1}, b_{2}, b_{3}, b_{i}, b_{N}\right\} \leq 1 \text { and }
\end{gathered}
$$

from equation (4.6) we get

$$
\begin{gathered}
\left|E_{k}^{n+1}\right| \leq b_{k}\left|E_{k+1}^{n}\right|+\left(1-2 b_{k}\right)\left|E_{k}^{n}\right|+b_{k}\left|E_{k-1}^{n}\right|+b_{k} \sum_{j=1}^{k-1} g_{j}\left|\left[E_{k-j+1}^{n}-2 E_{k-j}^{n}+E_{k-j-1}^{n}\right]\right|+\tau^{n} \\
\leq\left(b_{k}+\left(1-2 b_{k}\right)+b_{k}\right)\left\|E^{n}\right\|+\max _{1 \leq i \leq N-1}\left\|\tau^{M}\right\|
\end{gathered}
$$

where $\quad\left\|E^{n}\right\|=\max _{1 \leq M \leq n}\left|E_{k}^{n}\right|$ and $\sum_{j=1}^{k-1} g_{j} \leq 0$.

Therefore, from equation (4.7) we get

$$
\begin{aligned}
& \left|E^{n+1}\right| \leq\left\|E^{n}\right\|+\max _{1 \leq M \leq n}\left\|\tau^{M}\right\| \\
& \left\|E^{0}\right\|=0, \text { implies }\left\|E^{n}\right\|=0
\end{aligned}
$$

Therefore, from equation (4.8), we get

$$
\left|E^{n+1}\right| \leq \max _{1 \leq M \leq n}\left\|\tau^{M}\right\|
$$

Since $(h, \tau) \rightarrow(0,0)\left\|\tau^{M}\right\|=0,(1 \leq M \leq n)$ implies that

$$
\left|E^{n+1}\right| \rightarrow 0 \text { uniformly in } \Omega \text { as }(h, \tau) \rightarrow(0,0)
$$

$$
\therefore\left|E_{j}^{n}\right| \rightarrow 0 \text { uniformly in } \Omega \text { as }(h, \tau) \rightarrow(0,0)
$$

Hence proof.

\section{Numerical Solution:}

In this section, we obtain the approximated solution of space fractional soil moisture diffusion equation with initial and boundary conditions. To obtain the numerical solution of the space fractional soil moisture diffusion equation (SFSMDE) by the finite difference scheme, it is important to use some analytical model. Therefore, we present an example to demonstrate that SFSMDE can be applied to simulate behavior of a 
fractional diffusion equation by using Mathematica Software. We consider the following, dimensionless onedimensional space fractional soil moisture diffusion equation with suitable initial and boundary boundary conditions

$$
\begin{aligned}
& \frac{\partial \theta(x, t)}{\partial t}=D \frac{\partial^{\alpha} \theta(x, t)}{\partial x^{\alpha}}, 0<x<1,1<\alpha \leq 2, t>0 \\
& \text { initial condition: } \theta(\mathrm{x}, 0)=0,0 \leq x \leq 1 \\
& \text { boundary conditions : } \theta(0, \mathrm{t})=1, \\
& \theta_{x}(x, t)=0 \text {, as } x \rightarrow \infty, t>0
\end{aligned}
$$

with the diffusion coefficient $D=1$.

The numerical solution obtained at $t=0.05$ by considering the parameters $\tau=0.005, h=0.1$, $\alpha=1.7,1.8$ and 1.9 , which is simulated in the following figure.

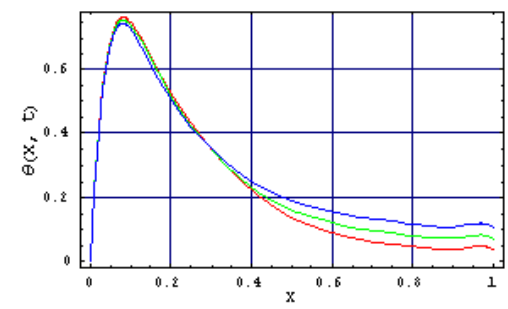

Fig.5.1 : The soil moisture diffusion profile with $t=0.05$, $h=0.1, \alpha=1.7$ (blue) $\alpha=1.8($ red $)$ and $\alpha=1.9$ (green)

\section{Conclusions.}

( i ) We develop the new space fractional order explicit finite difference scheme for soil moisture space fractional diffusion equation in a bounded domain.

(ii) The numerical example is presented to show that the numerical results are in good agreementwith our theoretical analysis.

(iii) The fractional order explicit finite difference scheme is numerically stable.

\section{References.}

[1] A. P. Bhadane, K. C. Takale, Basic Developments of Fractional Calculus and its Applications, Bulletin of Marathwada Mathematical Society, Vol. 12, No. 2, p. 1-17 (2011)

[2] Florica MATEL and BUDIU , Differential equations and their applications to the Soil Moisture Study, Bulletin UASVM, Horticulture 65(2)(2008).

[3] R. Hilfer, Applications of Fractional Calculus in Physics, World Scientific, Singapore (2000).

[4] S. M. Jogdand, K. C. Takale, V. C. Borkar, Fractional Order Finite Difference Scheme For Soil Moisture Diffusion Equation and its Applications, IOSR Journal of Mathematics(IOSR-JM), Volume 5, pp 12-18, 4 (2013).

[5] Daniel Hillel, Introduction to Soil Physics, Academic Press (1982).

[6] Don Kirkham and W.L. Powers, Advanced Soil Physics, Wiley-Interscience (1971).

[7] F.Liu, P. Zhuang, V. Anh, I, Turner, A Fractional Order Implicit Difference Approximation for the Space-Time Fractional Diffusion equation, ANZIAM J.47 (EMAC2005), pp. C48-C68:(2006).

[8] Pater A.C., Raats and Martinus TH. Ven Genuchten, Milestones in Soil Physics, J. Soil Science 171,1(2006). 10

[9] I. Podlubny, Fractional Differential equations, Academic Press, San Diago (1999).

[10] S. Shen, F.Liu, Error Analysis of an explicit Finite Difference Approximation for the Space Fractional Diffusion equation with insulated ends, ANZIAM J.46 (E), pp. C871-C887:(2005).

[11] K. C. Takale, V. R. Nikam, A. S. Shinde, Mittag-Leffleer Functions, its Computations and Applications to Differential Equations of Fractional Order, Mathematical Modeling and Applied Soft Computing, Vol. 1, p. 561-575 (2012).

[12] K. C. Takale, V. R. Nikam, A. S. Shinde, Finite Difference Scheme for Space Fractional Diffusion Equation with Mixed Boundary Conditions, American Jr. of Mathematics and Sciences, Vol. 2, No. 1, p. 291-295, (2013). 